\title{
New Theory of Stellar Convection without the mixing-length parameter: new stellar atmosphere models
}

\author{
Stefano Pasetto ${ }^{1}$, Cesare Chiosi ${ }^{2}$ and Mark Cropper ${ }^{1}$ \\ ${ }^{1}$ Mullard Space Science Laboratory, University College London, Holmbury, St. Mary, \\ Dorking, Surrey RH5 6NT, United Kingdom \\ email: s.pasetto@ucl.ac.uk \\ ${ }^{2}$ Dept. of Physics \& Astronomy "Galileo Galilei", University of Padua, Vicolo \\ dell'Osservatorio, 5, 35141 Padova PD, Italy
}

\begin{abstract}
Stellar convection is customarily described by the mixing-length theory, which makes use of the mixing-length scale to express the convective flux, velocity, and temperature gradients of the convective elements and stellar medium. The mixing-length scale is taken to be proportional to the local pressure scale height, and the proportionality factor (the mixing-length parameter) must be determined by comparing the stellar models to some calibrator, usually the Sun. No strong arguments exist to suggest that the mixing-length parameter is the same in all stars and all evolutionary phases. Because of this, all stellar models in the literature are hampered by this basic uncertainty.

In a recent paper (Pasetto et al. 2014) we presented a new theory that does not require the mixing length parameter. Our self-consistent analytical formulation of stellar convection determines all the properties of stellar convection as a function of the physical behavior of the convective elements themselves and the surrounding medium. The new theory of stellar convection is formulated starting from a conventional solution of the Navier-Stokes/Euler equations, i.e. the Bernoulli equation for a perfect fluid, but expressed in a non-inertial reference frame co-moving with the convective elements. In our formalism, the motion of stellar convective cells inside convective-unstable layers is fully determined by a new system of equations for convection in a non-local and time-dependent formalism.

We obtained an analytical, non-local, time-dependent solution for the convective energy transport that does not depend on any free parameter. The predictions of the new theory are compared with those from the standard mixing-length paradigm with positive results for atmosphere models of the Sun and all the stars in the Hertzsprung-Russell diagram.
\end{abstract}

Keywords. Sun: fundamental parameters Sun: interior stars: evolution stars: fundamental parameters

\section{Introduction}

The transfer of energy by convection is of paramount importance in all the stars. High-mass stars, roughly for masses $m>1.3 m_{\odot}$ contain fully convective cores, all stars $m \in\left[0.1,100\left[m_{\odot}\right.\right.$ have outer convective envelopes, and finally stars smaller in mass than $m<0.3 m_{\odot}$ are fully convective. Despite its great importance, a satisfactory treatment of stellar convection in stars is still open to debate and a self-consistent treatment of the physics of convective energy transfer is still missing.

In a recent paper Pasetto et al. (2014) developed the first theory of stellar convection in which the solar properties are reproduced without making use of free parameters. 

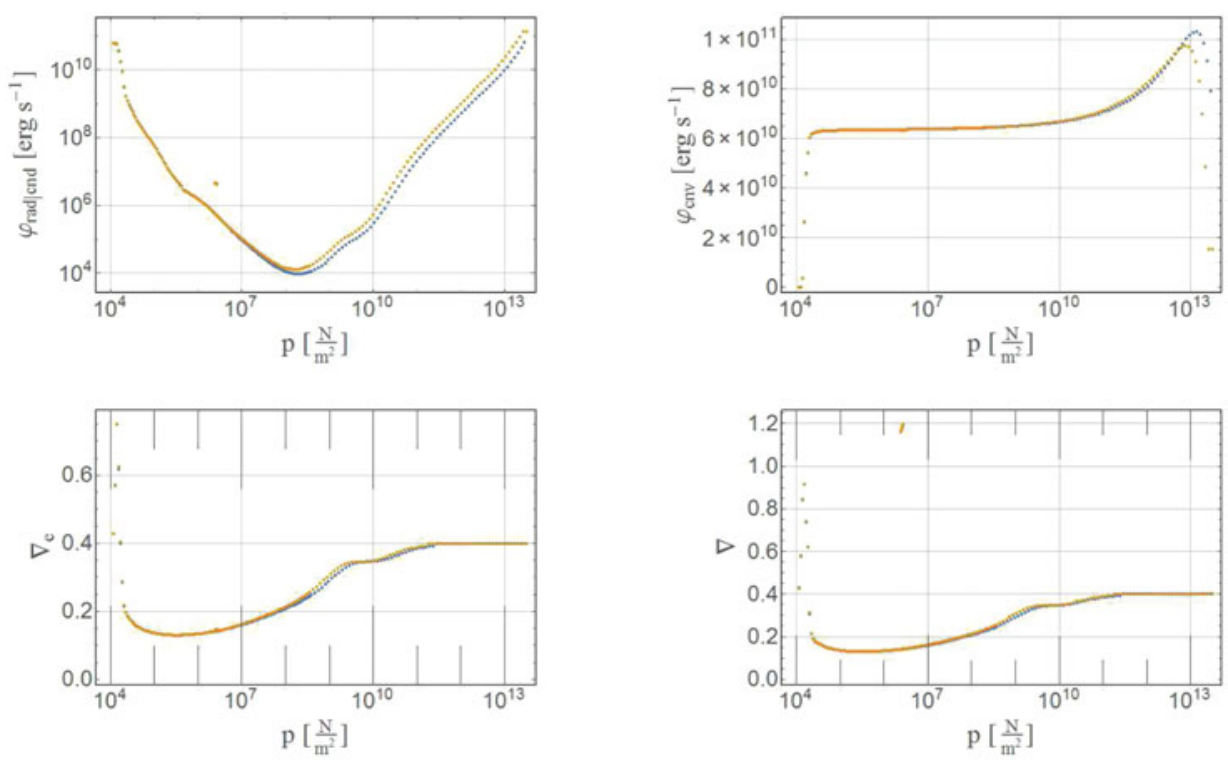

Figure 1. Solar fluxes and temperature gradient profiles for the internal pressure stratification of the star. The upper panels show the expectation for $\varphi_{\mathrm{rad} \mid \mathrm{cnd}}$ on the left and $\varphi_{\mathrm{cnv}}$ on the right. Yellow refers to our theory, blue to the MLT.

\section{Results: The model matching the Sun}

We present here a comparison between the standard MLT and the SFC theory. We consider the stellar track of Bertelli et al. (2008) best fitting the present position of the Sun on the HRD e.g., $\log _{10}\left\{L / L_{\odot}, T_{\text {eff }}\right\} \cong\{0.000,3.762\}$ with standard chemical composition $\{X, Y\}=\{0.71,0.27\}$. The results are shown in Fig.1. In the same plot we show also the predictions of the MLT with $\Lambda_{m}=1.65$ (the MLT is according to the version presented in Kippenhahn et al. (2012)), so that comparison between SFC theory and MLT is possible. Both the temperature gradients $\nabla$ and $\nabla_{e}$ and fluxes $\varphi_{\text {rad }} \mid$ cnd and $\varphi_{\text {cnv }}$ predicted by SFC theory and MLT are in mutual agreement over an impressive range in pressure of almost ten orders of magnitude.

\section{References}

Bertelli, G., Bressan, A., Chiosi, C., Ng, Y. K., \& Ortolani, S., 1994, Memorie della Societ Astronomia Italiana, 65, 689

Bertelli, G., Girardi, L., Marigo, P., \& Nasi, E., 2008, A\&A, 484, 815

Kippenhahn, R., Weigert, A., \& Weiss, A., 2012, Stellar Structure and Evolution

Pasetto, S., Chiosi, C., Cropper, M., \& Grebel, E. K., 2014, MNRAS, 445, 3592 